\title{
Deformed microcavities with very high Q-factors and directional farfield emis- sion
}

\author{
Arne Behrens ${ }^{1, *}$, Martí Bosch ${ }^{2,}$, Martina Hentschel ${ }^{3}$, and Stefan Sinzinger ${ }^{1,}$ \\ ${ }^{1}$ FG Technische OptikInstitut für Mikro- und Nanotechnologien MacroNano ${ }^{\circledR}$, TU Ilmenau \\ ${ }^{2}$ FG Computational Physics, TU Ilmenau \\ ${ }^{3}$ Intstitut für Physik, Technische Universität Chemnitz
}

\begin{abstract}
We report the design and optimized fabrication of deformed whispering gallery mode resonators in silica with solely ICP-RIE. This allows us to control the morphology of the resonators more freely and results in low surface roughness. The light was coupled into the resonator using a state of the art tapered fiber approach and we determined the Q-factor in the range of $10^{5}$
\end{abstract}

\section{Introduction}

Optical microcavities are structures that confine light to a defined volume using the mechanisms of reflection and resonance. A special type of modes inside a circular optical microcavity is called whispering gallery mode (wgm) because they are confined by the total internal reflection occurring at the cavity boundary. One main property of a resonator is the Q-factor, which describes the ratio of stored energy inside the resonator to the loss energy per cycle. A high Q-factor, therefore, means long photon lifetime inside the cavity and this increases the light-matter interaction drastically. This is the main reason why high$\mathrm{Q}$ resonators are a research platform for several fundamental investigations and experimental applications [1]. In the recent time high-Q resonators have been used for e.g. quantum electro dynamics [2] ,telecommunications [3], optomechanics, nonlinear optics [4] and sensing [5].

\section{Asymmetric resonant cavities}

One intriguing field of study are asymmetric resonant wgm cavities. While circular cavities exhibit a strongly isotropic radiation and propagation pattern, asymmetric resonant cavities show a more complex behavior. Much theoretical work was dedicated to the exploration of optical microcavities which produce a strongly directional farfield and to combine it with the property of high $\mathrm{Q}$ factors and therefore low loss [6].

Depending on the refractive indices of microresonstoars with specific deformations generate a directional far-field while still exhibiting high Q-factors. The limacon is such a morphology for refractive index larger than 3 [7]. Since most of the optical media with very low absorption have a refractive index close to 1.5 investigations

\footnotetext{
*e-mail: arne.behrens@tu-ilmenau.de
}

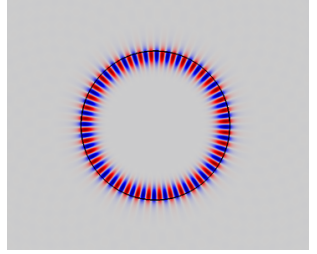

(a) Circle

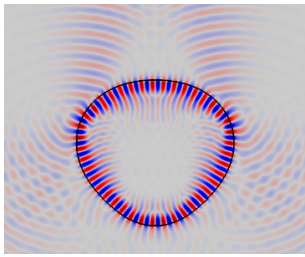

(b) Shortegg
Figure 1: Comparison of the mode structure

showed that a suitable morphology for this regime is the Shortegg, with a shape following the equation 2 .

$r(\phi)=R_{0}(1+0.16 \cos (\phi)-0.022 \cos (2 \phi)-0.05 \cos (3 \phi))$

\section{Fabrication}

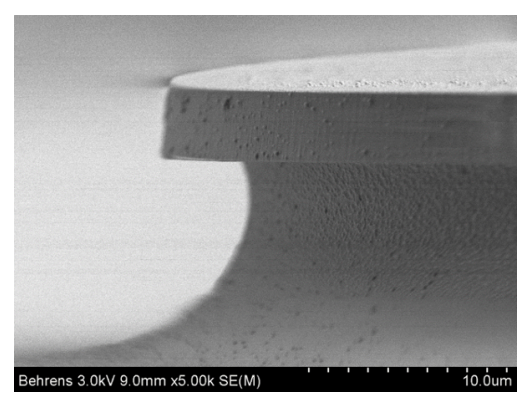

Figure 2: SEM image of a microdisk resonator

To investigate the behavior and potential applications of deformed cavities establish a fabrication process that allows us to fabricate resonators with very precise control of the deformation. The state of the art fabrication process 
for UHQ resonator was shown by [8]. They use buffered HF for the etching of the silica layer and underetch the silica disk with $\mathrm{XeF}_{2}$ vapour. After the structuring they use a reflow process to create a smooth boundary which results in a shrinkage of the resonator. First, we need to etch the $2 \mu \mathrm{m}$ thick silica layer, which is fabricated by thermal oxidation. For this, we use an inductively coupled plasma (ICP)-RIE with $\mathrm{CHF}_{3} / \mathrm{Ar}$ chemistry. By fine-tuning the etch gases and the ion energy it is even possible to adjust the slope angle of our resonator which allows us to confine mode in the z-direction. The following silicon underetching is achieved by using a cryogenic $S F_{6} / O_{2}$ ICP RIE which exhibits very high selectivity to silica which is critical for achieving a low surface roughness. We achieved a surface roughness of $R_{Q}=2 \mathrm{~nm}$.

\section{Experimental measurement}

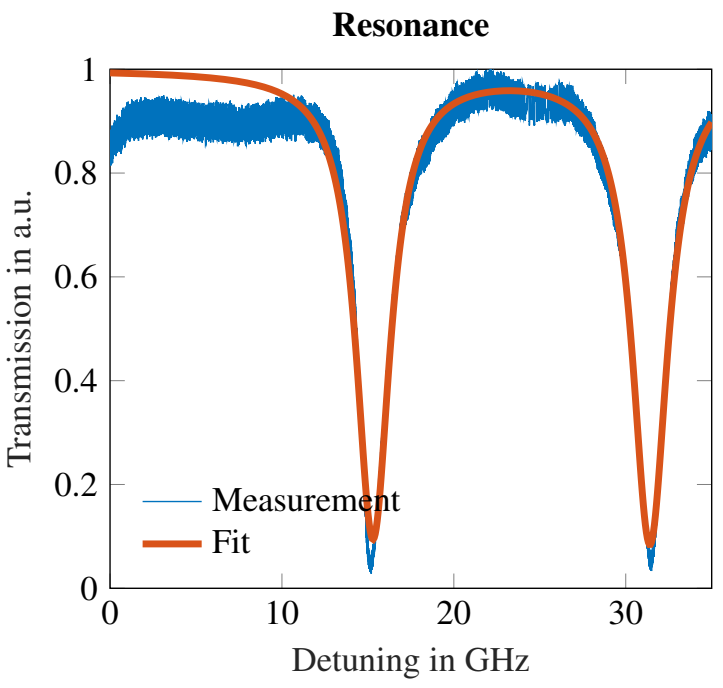

Figure 3: Fine scan of the resonance compared to a Lorentzian fit with a spectral window of $35 \mathrm{GHz}$

\section{$2 \mathrm{~cm}$}

For coupling light into the resonator, the most prominent method is the use of a so-called tapered fiber.

The shown measurements were conducted using a shortegg resonator with a diameter of $100 \mu \mathrm{m}$ or $k R=200$. The highest Q-factor measured was $10^{5}$ at a wavelength of about $1529 \mathrm{~nm}$ shown in 3 . The measurement data shows a good match with the simulated mode behavior. The divergence on the left side can be attributed to some low q modes. The fitted model consists of two Lorentzian peaks with the position $15.3 \mathrm{GHz}$ and $31.4 \mathrm{GHz}$ with an individual width of $2.3 \mathrm{GHz}$ and $2.5 \mathrm{GHz}$. This indicates the presence of mode splitting and mode broadening which could be caused by several pertubations like surface roughness, microparticles which are stuck to the surface or the presence of the tapered fiber itself.

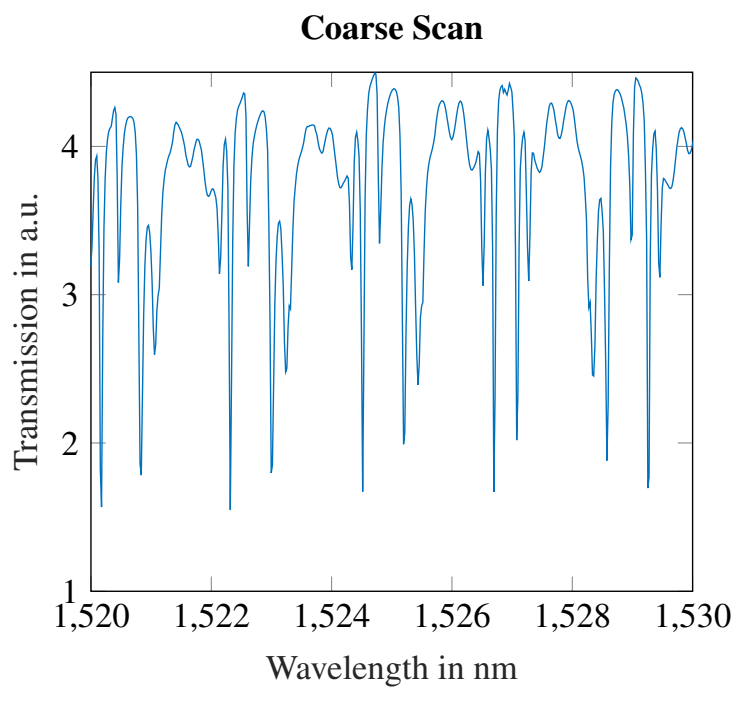

Figure 4: Measured resonances by monitoring the transmission of a tapered single mode fiber

\section{Simulation}

To evaluate the experimental measurements we designed a wave optical simulation in comsol. There we computed the eigenmodes of a $100 \mu \mathrm{m}$ Shortegg to compare them to our measurements. They resulted in a maximum Q-factor of $3 * 10^{6}$. However this is the maxium intrinsic Q-factor which could be achieved in the eigenmode evaluations.

\section{Conclusion}

We demonstrated the fabrication of High-Q using ICP-RIE which resulted in measured Q-factors in the range of $10^{5}$ in deformed cavities with a diamter of $100 \mu \mathrm{m}$.

\section{Acknowledgements}

I want to thank Prof. Síle Nic Chormaic for the possibily to visit the Light-Matter-Interaction Unit at OIST and to conduct measurements at their laboratory.

\section{References}

[1] K.J. Vahala, nature 424, 839 (2003)

[2] S. Spillane, T. Kippenberg, K. Vahala, K. Goh, E. Wilcut, H. Kimble, Physical Review A 71, 013817 (2005)

[3] T.A. Ibrahim, R. Grover, L. Kuo, S. Kanakaraju, L. Calhoun, P. Ho, IEEE Photonics Technology Letters 15, 1422 (2003)

[4] T.J. Kippenberg, R. Holzwarth, S.A. Diddams, science 332, 555 (2011)

[5] M.D. Baaske, M.R. Foreman, F. Vollmer, Nature nanotechnology 9, 933 (2014)

[6] J. Wiersig, M. Hentschel, Physical review letters 100, 033901 (2008)

[7] J. Kreismann, S. Sinzinger, M. Hentschel, Physical Review A 95, 011801 (2017) 